\title{
Molecular organization in MAPLE-deposited conjugated polymer thin films and the implications for carrier transport characteristics
}

\author{
Ban Xuan Dong, ${ }^{1}$ Anton Li, ${ }^{1}$ Joseph Strzalka, ${ }^{3}$ Gila E. Stein ${ }^{2}$ and Peter F. Green ${ }^{1, *}$ \\ ${ }^{1}$ Department of Materials Science and Engineering, Biointerfaces Institute, University of Michigan, Ann \\ Arbor, MI 48109, USA \\ ${ }^{2}$ Department of Chemical and Biomolecular Engineering, University of Houston, Houston, TX 77204, USA \\ ${ }^{3}$ Argonne National Laboratory, X-Ray Science Division, Argonne, Illinois, 60439, USA \\ Correspondence to: Peter F. Green (E-mail: pfgreen@umich.edu)
}

ABSTRACT: The morphological structure of poly(3-hexylthiophene) (P3HT) thin films deposited by both Matrix Assisted Pulsed Laser Evaporation (MAPLE) and solution spin-casting methods are investigated. The MAPLE samples possessed a higher degree of disorder, with random orientations of polymer crystallites along the side-chain stacking, $\pi-\pi$ stacking, and conjugated backbone directions. Moreover, the average molecular orientations and relative degrees of crystallinity of MAPLE-deposited polymer films are insensitive to the chemistries of the substrates onto which they were deposited; this is in stark contrast to the films prepared by the conventional spin-casting technique. Despite the seemingly unfavorable molecular orientations and the highly disordered morphologies, the in-plane charge carrier transport characteristics of the MAPLE samples are comparable to those of spin-cast samples, exhibiting similar transport activation energies ( $56 \mathrm{meV}$ versus $54 \mathrm{meV}$ ) to those reported in the literature for high mobility polymers.

KEYWORDS: MAPLE, conjugated polymers, X-Ray, ellipsometry, organic thin film transistor, structuretransport relationship.

\section{INTRODUCTION}

With their important applications including thin film transistors, ${ }^{1}$ lighting, displays $^{2,3}$ and organic solar cells, ${ }^{4,5}$ the molecular design, synthesis and processing of conjugated polymers is of significant scientific and technological interest. Charge carrier mobilities, and hence device performance, are strongly influenced by the morphology of conjugated polymers. ${ }^{6-8}$ Therefore, understanding the interrelations between chemistry, processing, morphology, and thus optoelectronic performance is crucial for designing high performance polymeric semiconductor materials.
Thin conjugated polymer films can be prepared by solution or vacuum-based deposition techniques. Whereas solution-based methods offer advantages of low-cost and highthroughput manufacturing, the fabrication of complex systems such as layered, nanopatterned structures, or in cases of underlying surfaces having poor wettability poses significant challenges. Recently, the vacuumbased deposition technique matrix-assisted pulsed laser evaporation (MAPLE) has been increasingly employed for fabrication of thin polymer films by different research groups. ${ }^{9-12}$ The film deposition process in MAPLE involves the absorption of light with a specific wavelength from a laser by a frozen dilute polymer/solvent mixture. Ideally, the sacrificial host solvent is chosen such that it absorbs the 
majority of the laser energy, thereby minimizing or altogether avoiding the photochemical degradation of the guest polymer. Together with its advantages of being a vacuum-based deposition technique, the MAPLE technique enables the production of films with unique morphologies and associated physical properties. $^{13,14}$ With its unprecedented capabilities, MAPLE has readily been exploited for growing thin polymer films for a wide range of applications including sensors, ${ }^{15,16}$ drug delivery and medical implants. ${ }^{17}$ Recently, promising findings have also been reported on the properties of MAPLE deposited conjugated polymer films for solar cells, organic light emitting diodes and other organic electronic applications. ${ }^{18-26}$ Although operational devices have been made, the detailed molecular structure and the fundamental connection between processing, morphology and transport in those devices are still poorly understood.

Here, using grazing-incidence wide angle X-ray scattering (GIWAXS), we investigate the structure of MAPLE-deposited P3HT films on various types substrates and compare the results to films produced using conventional spin-casting. Three common substrates for organic electronic devices were used: $\mathrm{SiO}_{2} / \mathrm{Si}$, octyltrichlorosilane (OTS)-treated $\mathrm{SiO}_{2} / \mathrm{Si}$, and poly(3,4-ethylenedioxythiophene) polystyrene sulfonate (PEDOT)/indium tin oxide (ITO)/glass. The GIWAXS studies, complemented by variable angle spectroscopic ellipsometry (VASE) measurements, reveal that MAPLE-deposited samples possess a higher degree of disorder, with more random orientations of polymer crystallites along side-chain stacking, $\pi-\pi$ stacking and conjugated backbone directions. Unlike solvent-cast films, the morphologies of the MAPLE-deposited films are independent of the substrate onto which they are deposited. Our studies provide important new insights into the mechanism of film formation of MAPLEdeposited semicrystalline conjugated polymer films in connection to in-plane charge carrier transport properties.

\section{EXPERIMENTAL}

Preparation of spin-cast and MAPLE samples: All substrates used in this study were cleaned by ultrasonication in an Alconox ${ }^{\circ}$ detergent solution, DI water, acetone, hot Hellmanex solution and 2-propanol for $5 \mathrm{~min}$ each, followed by UV-ozone treatment for 20 min. Thin film transistor (TFT) measurements were performed on polymer films deposited on highly doped Si with $300 \mathrm{~nm}$ of thermally-grown $\mathrm{SiO}_{2}$. GIWAXS measurements were performed on polymer films deposited on 3 different substrates: Si with $300 \mathrm{~nm}$ of thermally grown $\mathrm{SiO}_{2}\left(\mathrm{SiO}_{2} / \mathrm{Si}\right)$, octyltrichlorosilane (OTS)-treated $\mathrm{SiO}_{2} / \mathrm{Si}$ and indium tin oxide (ITO)/glass coated with a layer of poly(3,4ethylenedioxythiophene) polystyrene sulfonate (PEDOT). The self-assembled monolayer of OTS (Sigma-Aldrich) was grown on top of the $\mathrm{Si} / \mathrm{SiO}_{2}$ substrate by immersing the substrate in a mixture of OTS and hexadecane (1:250 by volume) for 14 hours while stirring. A smooth OTS layer was formed on top of the $\mathrm{Si} / \mathrm{SiO}_{2}$ substrate as confirmed by Atomic Force Microscopy (data not shown). PEDOT/ITO/glass substrates were fabricated by spin-casting filtered PEDOT solution (Clevios PH 500) onto the cleaned ITO/glass substrates and annealed at $130{ }^{\circ} \mathrm{C}$ for $20 \mathrm{~min}$ before polymer deposition. The ellipsometric measurements were performed on films supported by Si substrates possessing different thermal oxide layer thicknesses.

Solutions of P3HT (Rieke Metal, 95\% regioregularity, $M_{w}=50000 \mathrm{~g} \cdot \mathrm{mol}^{-1}$ ) were prepared by dissolving the polymer in 1,2dichlorobenzene with concentration $10 \mathrm{mg} / \mathrm{ml}$ and shaken overnight before filtering with a $0.45 \mu \mathrm{m}$ filter. The filtered solutions were then spun onto the prepared substrates at $600 \mathrm{rpm}$ for $2 \mathrm{~min}$ to make spin-cast films. For OTStreated substrates, the P3HT solutions were left to stand on the substrates for several minutes before spin-casting due to the poor wettability of OTS. 
Our MAPLE deposition system was purchased from PVD Products, equipped with an Er:YAG laser (Quantel) that produces a wavelength of $2.94 \mu \mathrm{m}$. We exploited an emulsion-based approach pioneered by the Stiff-Roberts's group. ${ }^{21}$ In this strategy, the polymer is first dissolved in 1,2-dichlorobenzene $(5 \mathrm{mg} / \mathrm{ml})$, then mixed with benzyl alcohol and deionized (DI) water (containing 0.005 wt.\% sodium dodecyl sulfate surfactant) at a 1:0.3:3 ratio, and then shaken and ultrasonicated to generate a homogeneous emulsion. The emulsion was injected into a pre-cooled target cup (ca. $-170{ }^{\circ} \mathrm{C}$ ); once it was fully frozen, the chamber was pumped in a high vacuum to a pressure $<2 \times 10^{-5}$ Torr. To maintain relatively uniform/consistent ablation over the course of deposition, the target was subjected to a constant rotation, while the laser (fluence $\sim 1.3$ $\mathrm{J} / \mathrm{cm}^{2}$ at a repetition rate of $5 \mathrm{~Hz}$ ) was rastered across the surface. The substrates were suspended in face-down at a height of $5.5 \mathrm{~cm}$ above the target, and were also kept at a constant rotation to achieve uniform deposition. The deposition time was approximately 5 hours. Film thicknesses of all spin-cast and MAPLE samples in this study were kept at ca. $80 \mathrm{~nm}$, as confirmed by both AFM and spectroscopic ellipsometry.

In-plane mobility measurements: Topcontact, bottom-gate transistor configurations were used to fabricate transistors for in-plane mobility measurements. Thin film transistors were made by depositing polymer on top of OTS-treated substrates. After deposition of polymer films, source and drain gold electrodes (Kurt J. Lesker, 99.99\%) were vacuum-deposited on top of the polymer film at a rate of $0.5 \mathrm{~A} / \mathrm{s}$ to fabricate transistors having channel length and width of $50 \mu \mathrm{m}$ and $500 \mu \mathrm{m}$, respectively. Inplane hole mobility $(\mu)$ was measured using the Agilent 4156C Parameter Analyzer. The hole mobility was extracted from the drain current $I_{D S}$ by fitting the transfer curve in the saturation regime $\left(V_{D}=-80 \mathrm{~V}\right)$ using the following equation:

$$
I_{D S}=\frac{W C_{i}}{2 L} \mu\left(V_{g}-V_{t}\right)^{2}
$$

In this equation, $W$ and $L$ are the channel width and length, $C_{i}=10 \mathrm{nF} / \mathrm{cm}^{2}$ is the capacitance per unit area of the insulating $\mathrm{SiO}_{2}$ layer, $V_{g}$ and $V_{t}$ are the gate and threshold voltage, respectively. The experiments were conducted in a vacuum cryostat (Janis Inc.) at specific temperatures controlled by a Temperature Monitor (LakeShore Cryotronics).

Grazing incidence wide angle X-ray scattering (GIWAXS): All samples were measured at beamline 8-ID-E of the Advanced Photon Source (Argonne National Laboratory) with $7.35 \mathrm{keV}(\lambda=0.16868 \mathrm{~nm})$ synchrotron radiation at an incidence angle of $0.21^{\circ}$, in ambient air. ${ }^{27}$ The off-specular scattering was recorded with a Pilatus $1 \mathrm{MF}$ pixel array detector (pixel size $=172 \mu \mathrm{m}$ ) positioned $204 \mathrm{~mm}$ from the sample. The measurement time was $10 \mathrm{sec}$ per frame, which is short enough to avoid damage to the structure, ${ }^{28}$ and data were acquired from 6 positions. Each data set was stored as a 981x1043 32-bit tiff image with 20bit dynamic range. The Pilatus detector has gaps along the horizontal axis that result in bands of missing data. To fill these gaps, the sample was moved to a fresh spot after each exposure, and measured again with the detector at a new vertical position. The gaps were filled by splicing the data from the two detector positions. This procedure is implemented using the GIXSGUI package for MATLAB. ${ }^{29}$ Signal-to-noise was improved by taking the sum of six data sets, which were output as intensity maps in $\left(q_{y}, q_{z}\right)$-space also by using the GIXSGUI package. The GIXSGUI package was used to correct the spectra for detection efficiency, the polarization effect and solid-angle variation.

In order to construct partial pole figures, wedge cuts with an angular breadth of $2^{\circ}$ were extracted from each GIWAXS data set for detector angles in the range of $90^{\circ}$ (vertical cut) up to $180^{\circ}$ (horizontal cut). Each wedge cut 
was fit to an empirical baseline function to subtract the background intensity. ${ }^{30}$ The integrated intensity of each peak is reported as a function of the polar angle $(\chi)$ between the scattering vector and pole vector. ${ }^{31}$ More details of partial pole figure construction can be found in the results and discussion section.

\section{Variable Angle Spectroscopic} Ellipsometry (VASE): VASE measurements were performed using the M-2000 Ellipsometer (J. A. Woollam Co.) on identical polymer films prepared on 4 different $\mathrm{Si}$ substrates. One substrate possessed a native layer of $\mathrm{SiO}_{2}$ and the other 3 possessed a layer of thermally grown $\mathrm{SiO}_{2}$; the thicknesses of each layer were 300, 500 and $750 \mathrm{~nm}$ (Encompass Inc.). VASE measurements were performed in the reflection mode at 5 angles: $55^{\circ}, 60^{\circ}, 65^{\circ}, 70^{\circ}$ and $75^{\circ}$. The complex reflectance ratio of the reflected and incident light polarization states are presented in terms of the ellipsometric angles $\psi$ and $\Delta$. The thickness and optical constants of P3HT were determined by iteratively fitting $\psi$ and $\Delta$ using the CompleteEASE software, also provided by J. A. Woollam Co. The optical constants of $\mathrm{Si}$, native oxide and thermally grown $\mathrm{SiO}_{2}$ were taken from CompleteEASE software's library database. The multi-sample analysis and interference enhancement methods were necessary to increase the uniqueness of the fits due to the strong correlations between the fitting parameters. ${ }^{32}$ More details of the fitting procedure can be found in the result and discussion section.

Atomic Force Microscopy (AFM): Topographical images of the top and buried interfaces of $\mathrm{P} 3 \mathrm{HT}$ films were probed using an Asylum Research MFP-3D stand-alone AFM in tapping mode with a CT300-25 Aspire probe (spring constant $40 \mathrm{~N} / \mathrm{m}$ and radius of curvature of $8 \mathrm{~nm}$ ). The buried interfaces were revealed by delaminating $\mathrm{P} 3 \mathrm{HT}$ films from the SAMtreated $\mathrm{SiO}_{2}$ substrates using a featureless poly(dimethyl siloxane) (PDMS) (Dow Corning Sylgard 184) stamp.

\section{RESULTS AND DISCUSSIONS}

This section is divided into two main parts. We begin by describing the structures of MAPLE and spin-cast films, determined by GIWAXS, VASE and AFM. We then discuss the role of structure on electronic transport, and the implications for film forming mechanism.

\section{GIWAXS}



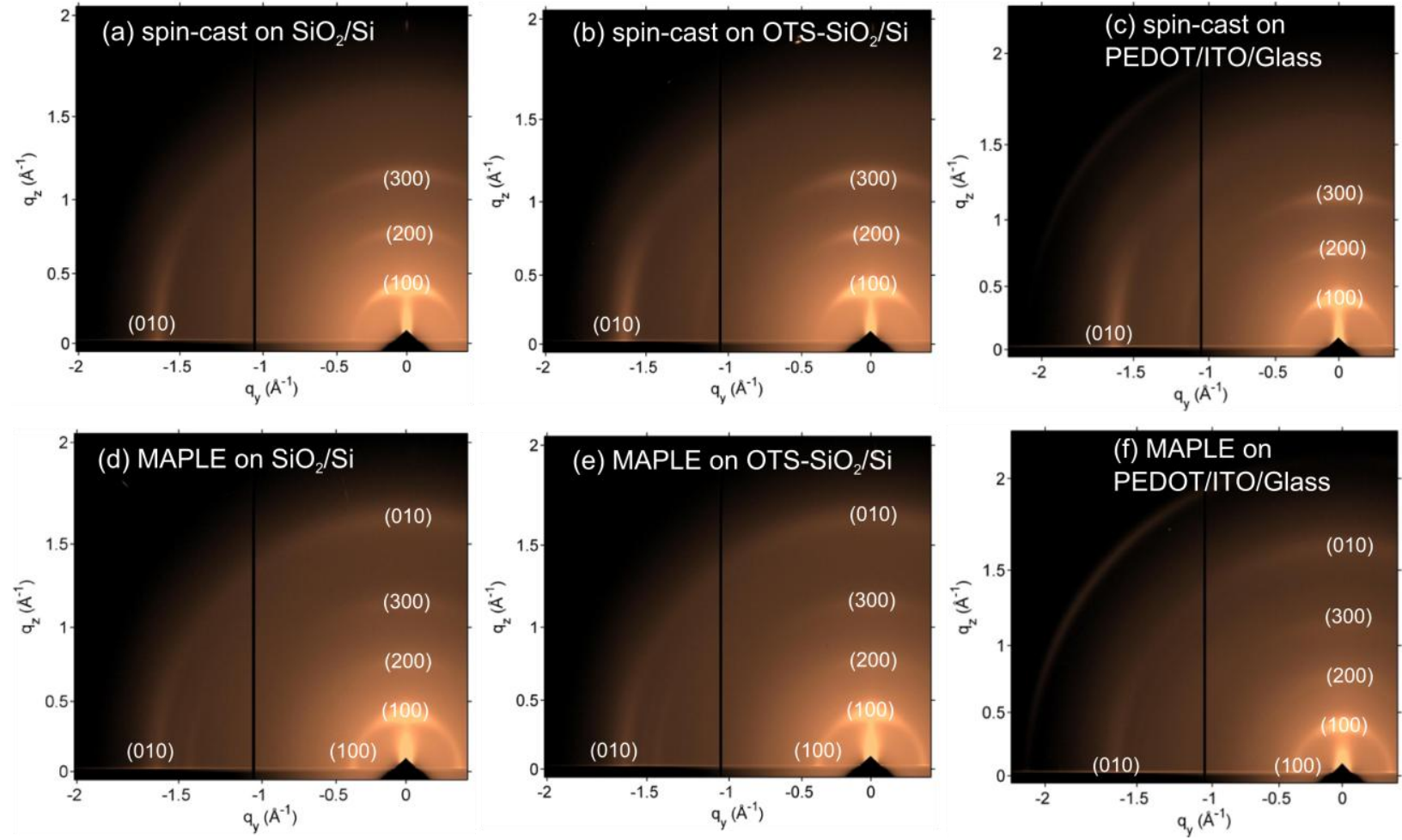

FIGURE 1: Diffraction patterns of (a),(b),(c) spin-cast and (d), (e), (f) MAPLE films deposited on $\mathrm{SiO}_{2} / \mathrm{Si}$, OTS-treated $\mathrm{SiO}_{2} / \mathrm{Si}$ and PEDOT/ITO/glass substrates.

GIWAXS experiments provide
information about the molecular packing
symmetry, lattice parameters, crystallite
orientation distributions, and relative degree of
crystallinity of MAPLE and spin-cast samples.
Shown in FIGURE 1 are GIWAXS patterns of
$\mathrm{MAPLE}$ and spin-casted films supported by
$\mathrm{SiO}_{2} / \mathrm{Si}$ OTS-treated $\mathrm{SiO}_{2} / \mathrm{Si}$ and
$\mathrm{PEDOT} / \mathrm{ITO} / \mathrm{glass}$ substrates; hereafter the
substrates will be denoted as $\mathrm{SiO}_{2}$, OTS and
PEDOT, respectively. The diffraction patterns of
films fabricated on different substrates using
the same technique are qualitatively similar. As

seen in FIGURE 1, for all spin-cast samples the (100) diffraction peak across the side-chain stacking direction is strongest along the out-ofplane $\left(q_{z}\right)$ direction, and the $(010)$ diffraction peak across the $\pi$-stacking direction is observed along the in-plane $\left(q_{\mathrm{xy}}\right)$ direction, indicating a strong bias towards the edge-on crystallite orientation. $^{33}$ In contrast, films prepared by MAPLE exhibit (100) and (010) diffraction peaks along both in-plane and out-of-plane directions, suggesting a more random distribution of polymer crystallite orientations. 
(a) OTS, out-of-plane

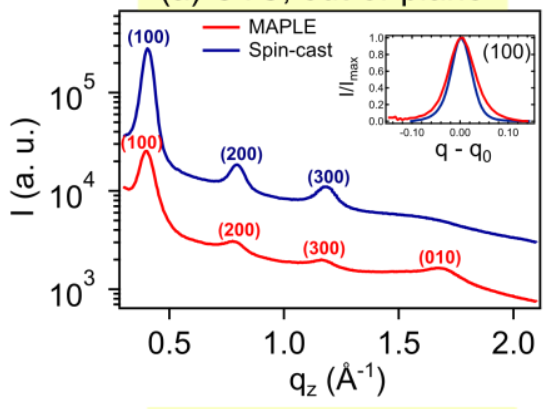

(d) OTS, in-plane

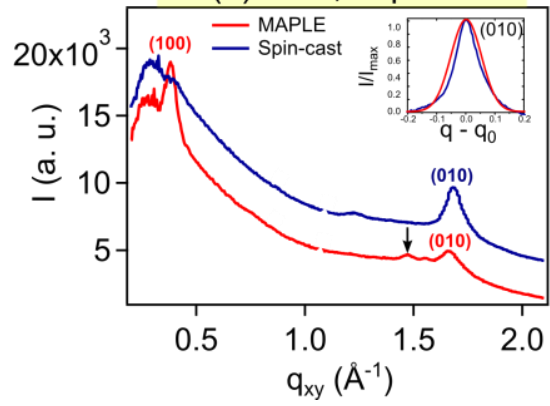

(b) $\mathrm{SiO}_{2}$, out-of-plane

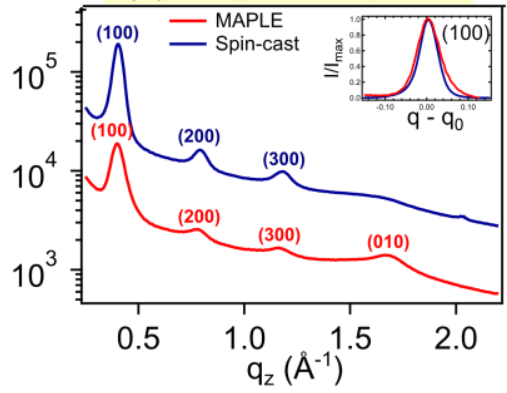

(e) $\mathrm{SiO}_{2}$, in-plane

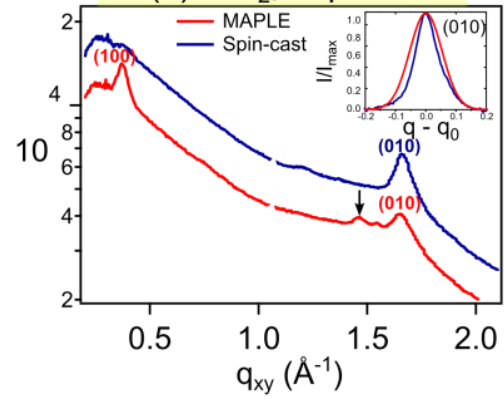

(c) PEDOT, out-of-plane

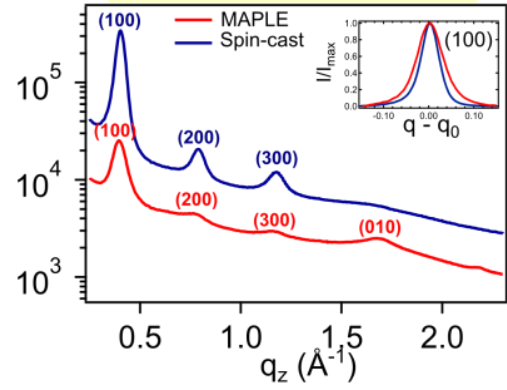

(f) PEDOT, in-plane

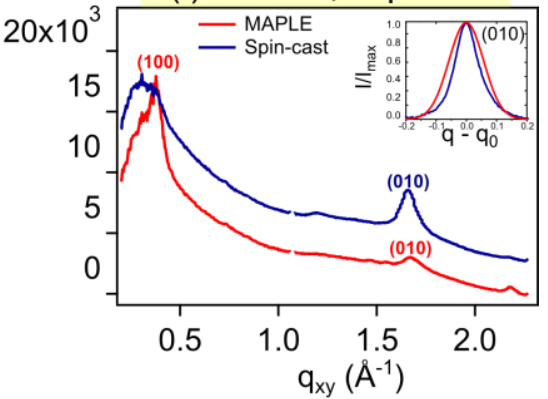

FIGURE 2: (a)(b)(c) Out-of-plane (vertical) and (d)(e)(f) in-plane (horizontal) diffraction signals of MAPLE and spin-cast samples on 3 different substrates. The diffraction intensity of MAPLE and spin-cast samples in each plot are offset vertically for clarity. Insets of $(a)(b)(c)$ and $(d)(e)(f)$ show superimposed line shapes of (100) and (010) diffraction peaks of MAPLE and spin-cast films on the corresponding substrates. Line shapes of (100) peaks were taken from the vertical line cuts, line shapes of (010) peaks were taken from the horizontal line cuts. The (010) diffraction peaks of MAPLE samples were calculated via multi-peak fitting to subtract the diffraction intensities of the adjacent peaks.

Figure 2 reports the intensity profiles along the out-of-plane $\left(q_{z}\right)$ and in-plane $\left(q_{x y}\right)$ directions. The out-of-plane intensity profiles were extracted from a detector angle of $\omega=0$ 으 (vertical). The in-plane intensity traces were measured at a detector angle of $\omega=83 \circ$ (nearly horizontal) instead of 90 ㅇ (horizontal), because at 90 o the data are noisier and partially obscured due to standing waves. Similar to visual inspection of the raw data, the intensity traces reveal clear differences in crystallite orientations between MAPLE and spin-cast films. For the spin-cast films, the (100) and (010) peaks are detected along the out-of-plane and in-plane axes, respectively, which indicates the edge-on crystallite orientations. On the other hand, for the MAPLE-deposited films, the (100) and (010) reflections appear along both directions indicating a more random orientation of crystallites. Furthermore, as shown in the insets of FIGURE 2, the (100) and (010) line shapes appear to be broader for the MAPLEdeposited samples than for the spin-cast samples; this is indicative of a shorter crystal coherence length both in the side-chain and $\pi$ stacking directions. We note that the crystal coherence length here is not exactly equal to the crystallite dimension because factors such as paracrystallinity could also contribute to the broadening of the diffraction peaks. ${ }^{34,35}$ Identifying the exact origins of the peak broadening requires more sophisticated lineshape analysis ${ }^{34,36}$ which is beyond the scope of our study. Nevertheless, we suspect that the broadening of diffraction peaks in MAPLEdeposited samples compared to spin-cast samples at least partially originates from the greater disordered morphology as suggested by AFM and UV-vis absorption spectroscopy 
measurements, reported earlier from our previous study. ${ }^{18}$

The peak positions $\left(q_{\text {exp }}\right)$ observed in both MAPLE and spin-cast samples are summarized in Table 1. Within experimental error, the peak positions are identical for all MAPLE and spin-cast samples deposited on all substrates. Interestingly, together with the commonly observed ( $h 00)$ and $(0 k 0)$ diffraction peaks, we also observe a weak peak at ca. $1.5 \AA^{-}$ 1 in the $q_{x y}$ direction of MAPLE samples, indicated by the arrows in FIGURE 2(d) and (e). This feature may be a mixed-index peak, which is consistent with a model based on a monoclinic unit cell as described by others. ${ }^{37,38}$ The monoclinic unit cell has lattice parameters of $a=16.1 \AA$, $b=7.6 \AA$, $c=7.6 \AA$, corresponding to the lamellar stacking axis, $\pi$-stacking axis and backbone direction, respectively. The typical angles for this monoclinic model of P3HT are $\alpha$ $=B=90^{\circ}$ and $\gamma=93.5^{\circ}$. (The angle $\gamma$ is between $a$ and $b$ axes). ${ }^{37}$ Table 1 provides a comparison of the observed and predicted peak positions. We index the peak at ca. $1.5 \AA^{-1}$ in MAPLE samples as a (310) reflection based on this monoclinic cell. It should nevertheless be emphasized that this assignment is very tentative, and more mixed index peaks are needed to verify the model. However, it is important to note that the mixed index peaks are observed exclusively in MAPLE samples in this work and only reported in a few literature studies on $\mathrm{P} 3 \mathrm{HT} .{ }^{37-40}$ Although there were some examples of mixed index peaks observed in spin-cast P3HT samples, ${ }^{39,40}$ they were all fabricated using chloroform, a solvent known to produce highly disordered morphology due to its high volatility. ${ }^{41}$ The appearance of mixed index peaks solely in MAPLE-deposited samples thus might suggest higher degree of chain folding and twisting in P3HT films deposited by MAPLE technique compared to those deposited by the spin-cast analog. ${ }^{42}$

TABLE 1: Summarized peaks position observed on MAPLE and spin-cast samples together with predicted peak positions based on the monoclinic unit cell with $a=16.1 \AA, b=7.6 \AA$, $c$ $=7.6 \AA, \alpha=6=90^{\circ}$ and $\gamma=93.5^{\circ}$.

\begin{tabular}{|l|l|l|l|}
\hline$(h k l)$ & $\begin{array}{l}q_{\text {exp }}\left(\AA^{-1}\right), \text { spin- } \\
\text { cast }\end{array}$ & $\begin{array}{l}q_{\text {exp }}\left(\AA^{-1}\right), \\
\text { MAPLE }\end{array}$ & $q_{\text {pred }}\left(\AA^{-1}\right)$ \\
\hline$(100)$ & 0.39 & 0.39 & 0.39 \\
\hline$(200)$ & 0.78 & 0.78 & 0.78 \\
\hline$(300)$ & 1.18 & 1.18 & 1.17 \\
\hline$(010)$ & 1.67 & 1.67 & 1.67 \\
\hline$(002)$ & 1.67 & 1.67 & 1.66 \\
\hline$(310)$ & Not observed & 1.48 & 1.48 \\
\hline
\end{tabular}

To quantify the molecular orientation distribution and relative degree of crystallinity (DoC) of all samples, partial pole figures of (100) reflections were constructed as a function of the polar angle $\chi$ between the scattering vector $q_{100}$ and the substrate normal (FIGURE 3(a)). In GIWAXS measurement, the detector angle $\omega$ is not exactly equal to the polar angle $\chi$ because the Ewald sphere is curved, as pointed out previously. ${ }^{31,43,44}$ The polar angle for the (100) reflection was calculated from each detector angle using the GIXSGUI package. ${ }^{29}$ The integrated intensity at each polar angle was scaled by $\sin (\chi)$ to correctly quantify the population of crystallites with a particular orientation. ${ }^{31,44}$ Shown in FIGURE 3(b) and (c) are the geometrically corrected partial pole figures of (100) reflection of spin-cast and MAPLE-deposited samples on 3 different substrates. The range of polar angles $\chi$ accessible with our experimental configuration is $3.2^{\circ}$ up to approximately $85^{\circ}$ for the (100) reflection. Data at higher angles are impacted by standing waves and thus excluded from the analysis, while data are lower angles cannot be resolved since the reciprocal lattice vector does not intersect with the Ewald sphere in this regime. $^{31}$ 
(a)

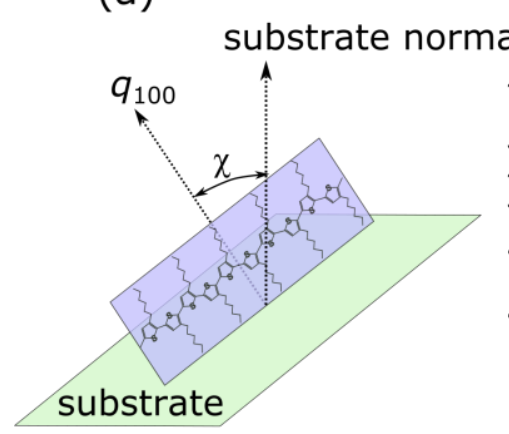

(b) spin-cast

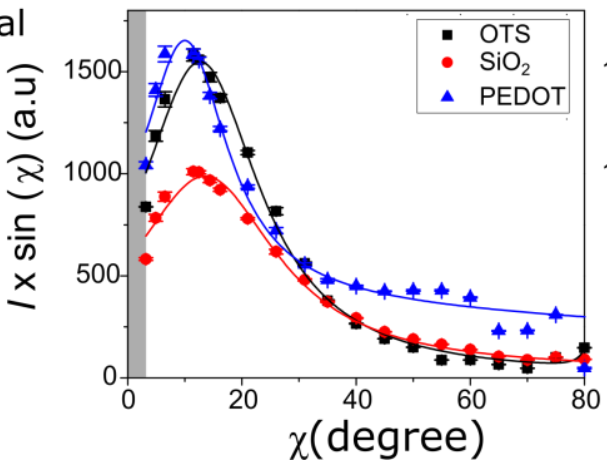

(c) MAPLE

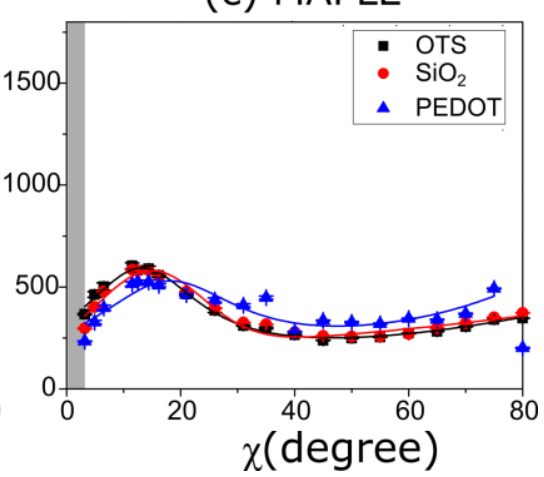

FIGURE 3. (a): Illustration of the polar angle $\chi$, defined as the angle between the substrate normal and the scattering vector along the side-chain direction $q_{100}$. (b), (c): geometrically corrected pole figures for (100) reflection of spin-cast and MAPLE samples deposited on three different substrates. The grayshaded areas in figure (b) and (c) denote the non-measurable regime of the experiments. The error bars are calculated from a propagation of errors approach that includes uncertainty in baseline correction and peak integration. The solid lines represent the best fits of the data to an empirical function. The axis scale of (b) and (c) plots are set to the same range for ease of comparison.

For P3HT films deposited by both techniques, pole figures of samples deposited on PEDOT/ITO/glass substrates appear to be noisier than those on $\mathrm{SiO}_{2}$ and OTS-treated $\mathrm{SiO}_{2}$ substrates, likely due to the comparative roughness of the PEDOT/ITO surfaces. All spincast samples exhibit a large population of crystallites with average orientation near $\chi=0$, which is consistent with an edge-on orientation where alkyl side-chains are nearly perpendicular to the substrate. In stark contrast, MAPLE samples exhibit a broad population of crystallite orientations with a very weak bias towards $\chi=$ 0ㅇ (edge-on) and $\chi=90$ o (face-on). This outcome agrees with visual inspection of raw detector images in FIGURE 1 and horizontal and vertical line cuts shown in FIGURE 2 . Furthermore, while samples spun on OTS and PEDOT exhibit larger edge-on populations than samples spun on $\mathrm{SiO}_{2}$, as evidenced by higher intensity toward $\chi=0$, there are no significant differences in the orientation distributions of the (100) reflections across the MAPLEdeposited counterparts. These results suggest that the average structure in spin-cast films is highly sensitive to the substrate chemistry, whereas the average structure in MAPLE deposited films is relatively independent of substrate chemistry.
In order to permit a comparison of the degrees of crystallinity (DoC) of the samples, a series of samples having the same thicknesses $h$ $\sim 80 \mathrm{~nm}$, confirmed by both ellipsometry and AFM, were prepared. X-ray exposure time and beam footprint size were made the same for all samples in order to further facilitate accurate DoC comparison. ${ }^{31}$ The pole figures were fitted using two Lorentzian functions centered near $\chi$ $=15^{\circ}$ and $\chi=90^{\circ}$, corresponding to diffraction from edge-on and face-on populations, respectively. Assuming that the data may be smoothly extrapolated to $\chi=0$ o and 90 ㅇ, integrating the intensity for the whole range of $\chi$ demonstrates that relative $\mathrm{DoC}$ of spin-cast samples is 1.6:1.3:1 for PEDOT, OTS, and $\mathrm{SiO}_{2}$, respectively. However, the DoC is identical within experimental uncertainty for MAPLE films deposited on the three substrates. Similar to the distributions of crystallite/aggregate orientations, the DoC also exhibits distinct substrate dependent behaviors, based on the fabrication techniques. This result is consistent with our previously reported findings that the in-plane mobility in MAPLE-deposited transistor devices is not as sensitive to the substrate chemistry as their spin-cast counterparts. ${ }^{18}$ 
Some limitations of our analysis should be noted. First, GIWAXS measures the average structure throughout the thickness of the film, so it is possible that crystallite orientations and DoC at the buried interface of MAPLE-deposited films may differ from the bulk. Second, because data for $\chi<3.2^{\circ}$ could not be resolved, crystallites with smaller polar angles are undetectable within our experiment set-up, as indicated by the gray-shaded areas in FIGURE $3(b)$ and (c). Therefore, some of the very highly edge-on oriented crystallites in spin-cast film on OTS substrate might have not been captured, ${ }^{45}$ so it is possible that the DoC of this sample was underestimated. However, such highly oriented crystallites in MAPLE samples are unlikely because of the highly disordered structure in MAPLE samples, evidenced by (i) heterogeneous globular morphology, ${ }^{18}$ (ii) broad distribution of conjugation length ${ }^{18}$ and (iii) random orientation of polymer chains determined by both GIWAXS and VASE measurement, as will be discussed in the following.

\section{Variable angle spectroscopic ellipsometry measurement}

Spectroscopic ellipsometric measurements were performed to determine the average orientation of the conjugated polymer backbones with regard to the underlying substrates. For all MAPLE and spincast samples, the best fits were achieved by employing the uniaxial anisotropic model, which assumes different dielectric functions for the in- and out-of-plane direction, but no preferred orientation within the $x y$ plane, i.e. $\varepsilon_{x x}^{\prime \prime}=\varepsilon_{y y}^{\prime \prime} \quad$ (in-plane) $\neq \varepsilon_{z z}^{\prime \prime}$ (out-of-plane). No significant differences were observed between the complex permittivities of P3HT films fabricated by the same technique on the three substrates. The representative imaginary parts of complex permittivity $\varepsilon^{\prime \prime}$ of MAPLE and spincast samples on $\mathrm{SiO}_{2}$ substrate are plotted in FIGURE 4.

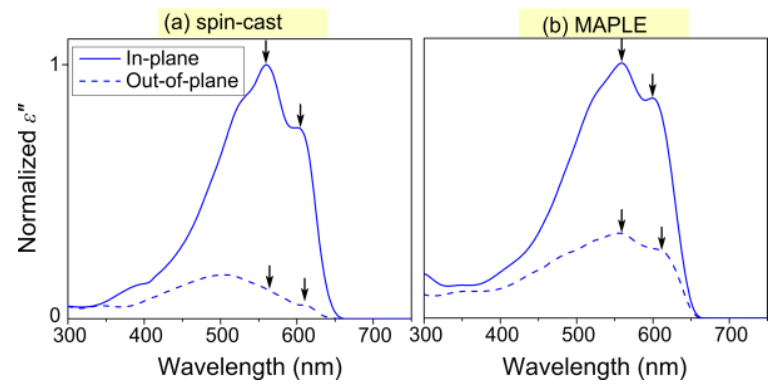

FIGURE 4: In-plane and out-of-plane imaginary permittivities $\varepsilon^{\prime \prime}$ of (a) spin-cast and (b) MAPLE films. The arrows indicate the aggregate shoulders in P3HT film.

It is evident that the shape of the inplane component in MAPLE-deposited film is less defined than that for spin-cast film, indicating a wider distribution of conjugation lengths in MAPLE-deposited sample. This is consistent with our previously reported finding using UV-vis absorption spectroscopy. ${ }^{18}$ Both samples exhibit pronounced anisotropic behavior with stronger in-plane than out-ofplane imaginary permittivities. This is indicative of the tendency of the polymer chains to lie parallel to the substrates, which has been widely observed in spin-cast conjugated polymers. $^{35,46,47}$ The MAPLE-deposited sample, however, exhibits a relatively stronger out-ofplane component as compared to the spin-cast counterpart, suggesting that the polymer chains in MAPLE samples are oriented more randomly. To quantify the orientation of the polymer chains, we compute the dichroic ratio $R$, defined as the ratio of the out-of-plane to the in-plane imaginary permittivity at $c a .610 \mathrm{~nm}(R$ $\left.=\varepsilon^{\prime \prime}{ }_{\text {out-of-plane }} / \varepsilon^{\prime \prime}{ }_{\text {in-plane }}\right)$; this position corresponds to the $\pi-\pi^{*}$ transition dipole moment in $\mathrm{P} 3 \mathrm{HT} .{ }^{47}$ The dichroic ratio $R$ provides a measure of the average orientation of the polymer backbone, where $R=1$ corresponds to a completely isotropic sample and $R=0$ corresponds to a film in which all the polymer chains lie parallel to the substrate. The observed dichroic ratio of spin-cast P3HT film is 0.12 , suggesting a predominantly in-plane orientation of polymer chains in spin-cast films. The dichroic ratio of the MAPLE-deposited film 
is 0.41 , which is significantly higher than that of spin-cast films and comparable to the value reported for spin-cast amorphous films. ${ }^{47}$ Interestingly, while the shape of $\varepsilon^{\prime \prime}$ in-plane and $\varepsilon^{\prime \prime}{ }_{\text {out-of-plane }}$ spectra appears similar for MAPLE sample, those of spin-cast samples differ significantly. In spin-cast samples, the vibronic shoulders that corresponding to aggregate absorption of $\mathrm{P}_{3} \mathrm{HT}^{48}$ (indicated by the dashed arrows in FIGURE 4(a)) are considerably suppressed in the out-of-plane direction, suggesting that the microstructure corresponding to the out-of-plane direction is much more disordered than the one corresponding to the in-plane direction. The resemblance of in- and out-of-plane spectra of MAPLE samples on the other hand indicates that the structure in the in- and out-of-plane directions are similar. ${ }^{49}$

\section{AFM}
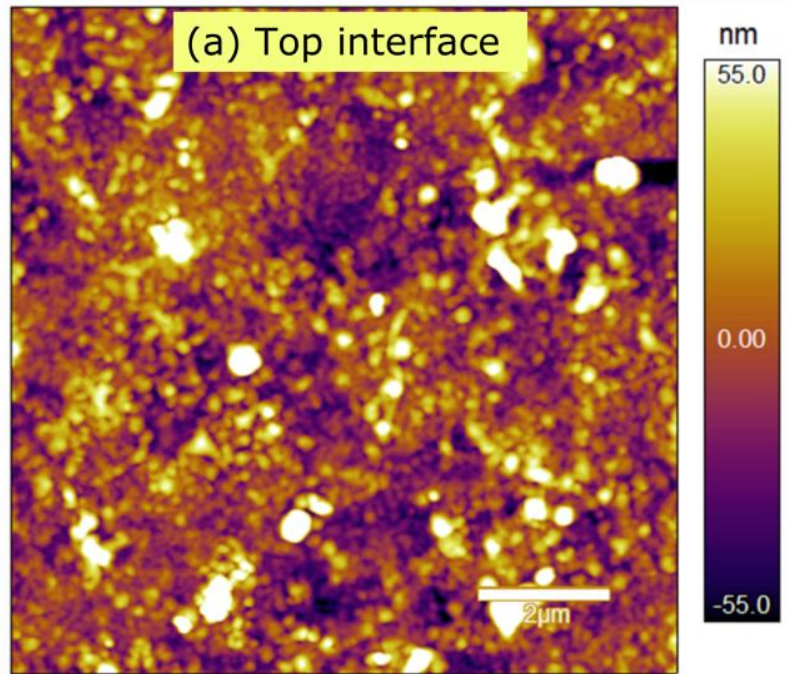

FIGURE 5: $10 \times 10 \mu \mathrm{m}$ AFM images of MAPLE-deposited P3HT's (a) top and (b) bottom (buried) interfaces. The samples were deposited on top of an OTS-treated substrate. The scale bar is $2 \mu \mathrm{m}$. The roughness for top and bottom interface is $24 \mathrm{~nm}$ and $13 \mathrm{~nm}$, respectively.

Because GIWAXS and VASE measure only the average morphologies across the entire film thickness, AFM measurements were performed to characterize the morphologies at the top and bottom interfaces of MAPLEdeposited samples. Shown in FIGURE 5(a) is the top surface of a MAPLE-deposited P3HT films on an OTS-treated $\mathrm{SiO}_{2}$ substrate. The surface of the film exhibits a roughness of ca. $24 \mathrm{~nm}$, consisting of globular features with diameters ranging from 10 to $200 \mathrm{~nm}$. This type of rough and inhomogeneous globular morphology is consistent with previous reports of MAPLE films, originating from the mechanisms of target ablation causing the polymer and solvent clusters to be ejected towards the substrate. ${ }^{50}$
Surprisingly, the AFM scan of the flipped film (substrate interface), shown in FIGURE 5(b), exhibits a very different morphology - no globular structures were observed and the surface is smoother, with a roughness of $c a .13$ $\mathrm{nm}$. Similar observations were made of MAPLEdeposited films on $\mathrm{SiO}_{2}$ (data not shown). This is not surprising since the polymer clusters that come in contact with the hard substrate are highly compliant. They may "crash" onto the hard substrates upon arrival and the force of impact resulting in flattened features observed in the AFM images of the flipped films.

\section{Implication of morphology on transport characteristics}


(a) spin-cast

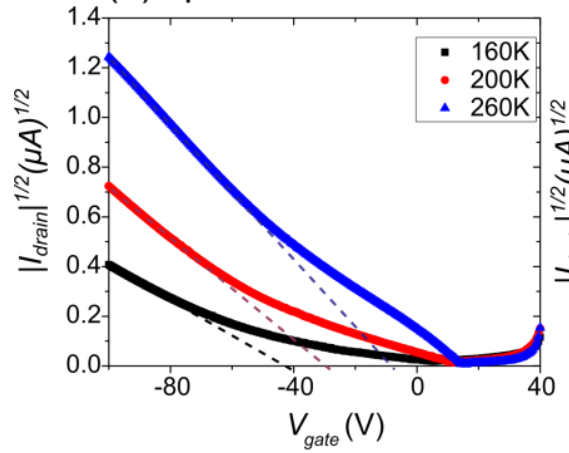

(b) MAPLE

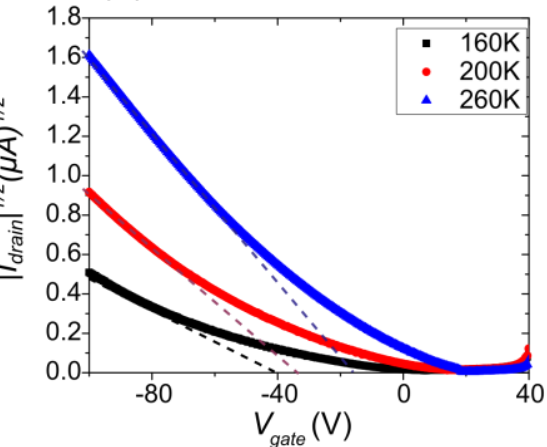

(c)

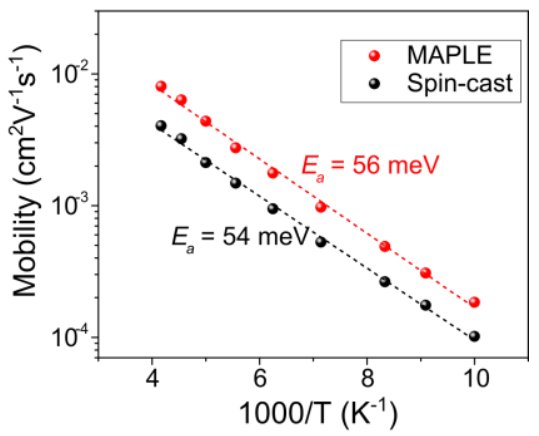

FIGURE 6: (a)(b) Exemplar transistor transfer characteristics of spin-cast and MAPLE fabricated samples, measured in saturation regime $\left(V_{\text {drain }}=-80 \mathrm{~V}\right)$ at three different temperatures are shown here. The dashed lines represent fits to the linear regime from which the in-plane mobilities are calculated. (c) Arrhenius plots of in-plane mobilities of both MAPLE and spin-cast samples. The transport activation energies $E_{a}$ are reported in the plot.

In order to understand the role of the morphology of the film on carrier transport, we measured the temperature dependence of inplane carrier mobility by fabricating bottomgate top-contact thin film transistors (TFTs) on OTS-treated substrates. FIGURE 6(a) and (b) depict the transistor transfer characteristics in the saturation regime $\left(V_{\text {drain }}=-80 \mathrm{~V}\right)$ at three different temperatures of spin-cast and MAPLE samples, respectively. It is apparent that for both samples, the drain current rises with increasing temperature, suggesting higher carrier mobilities at higher temperature. This is indicative of thermally activated charge transport characteristics typically observed in organic semiconductors. Despite the seemingly unfavorable molecular orientation (less in-plane orientation of the backbone and $\pi-\pi$ stacking) and the highly disordered morphology, the MAPLE-deposited sample exhibits very similar field-effect transport properties to the spin-cast sample with comparable or even superior inplane carrier mobilities at all temperatures within the range tested, as shown in FIGURE $6(c)$. The activation energies $E_{a}$ calculated from the Arrhenius equation are 56 and $54 \mathrm{meV}$ for MAPLE and spin-cast samples, respectively. These numbers are close to the transport activation energies of many high performing polymers used in TFT in literature such as pBTTT, high molecular weight P3HT, as well as many other high mobility donor-acceptor copolymers. ${ }^{35,51,52}$ This implies that TFT transport in both MAPLE-deposited sample and spin-cast sample, despite their morphological dissimilarity, might be limited by the same process that governs the activation energies.

The reasons for the comparable mobilities may be better understood from the following. First, GIWAXS and VASE measurements are only sensitive to the morphology of the bulk and therefore do not reflect the morphology near the buried interface where carrier transport in TFT transistors is known to occur. ${ }^{53}$ It could be that in MAPLE samples, the molecular order near the substrate is more favorable for in-plane transport than the bulk. We showed evidence that the topography of the polymer layer within the close vicinity of the dielectric interface is significantly different from the top. This layer may consist of aggregates with the conjugated backbone oriented parallel to the substrate or aggregates with strong edge-on orientations. An alternative explanation is that the absence of significant long-range order or highly oriented aggregates might not necessarily be detrimental for carrier transport, which has been increasingly observed in many recently engineered high mobility polymers ${ }^{35,54,55}$ In other words, the transport may be somewhat 
resilient to the structure. Due to the lack of long-range order, macroscopic charge migration in conjugated polymer films relies on the combination of fast transport of carriers along polymer backbone (intrachain transport) and relatively slower hopping transport across $\pi$ stacking direction (interchain transport). It was recently proposed that efficient long-range charge transport across conjugated polymer films only requires short-range intermolecular aggregation of a few polymer chains. ${ }^{56,57}$ As discussed in our previous publication, despite the very different morphologies, the UV-vis absorption spectrum and the extracted conjugation lengths of MAPLE-deposited film are very similar to those of spin-cast films. ${ }^{18}$ This suggests that at a very local scale, the chain aggregation behavior in MAPLE samples is similar to spin-cast samples. Here, such local aggregation is demonstrably sufficient for facile carrier transport in highly disordered MAPLE samples.

\section{Implication of morphology on film forming mechanism}

Unlike spin-cast samples, the average molecular orientation and DoC of MAPLEdeposited P3HT samples appear independent of the substrate chemistry, suggesting that different mechanisms drive the film formation compared to solvent-casting techniques. Both experiments and simulations suggest that film growth in MAPLE involves 3 different steps: (i) ejection of plumes of solvent-polymer droplets due to explosive decomposition of the solvent molecules after absorbing the energy from the laser pulses, (ii) rapid removal of solvent molecules from the solvent-polymer droplets during transport from target to substrate and (iii) deposition of the remaining polymer globules onto the substrate. ${ }^{50,58,59}$ Therefore, the formation of P3HT aggregates may occur during two distinct stages in the deposition process. In one case, they may form prior to arrival at the substrate, either in the frozen target or during the transfer from target to substrate. In another case, the aggregates may form subsequent to deposition of P3HT globules atop the substrate. To date, the exact origin and mechanism of aggregates formation in MAPLEdeposited conjugated polymer films remains elusive.

Based on our results, we hypothesize that most of the aggregates form prior to deposition at the substrate. This hypothesis is supported by several key observations. First, the DoC and orientation of the MAPLE films are independent of the substrate chemistry, suggesting that there is little or no nucleation, aggregation, or crystallization after the globules arrive at the substrate. Second, the random orientation of polymer aggregates in the films suggests there is little interaction with the substrate during aggregate formation. This assumption is further corroborated by the line shape of $\varepsilon^{\prime \prime}{ }_{\text {in-plane }}$ and $\varepsilon_{\text {out-of-plane }}$ obtained from VASE measurement (FIGURE 4). For spin-cast samples, the aggregate shoulders in $\varepsilon^{\prime \prime}$ out-of-plane are considerably weaker than those in $\varepsilon^{\prime \prime}{ }_{\text {in-plane }}$, revealing significant chain disorder in the outof-plane directions. This reflects the preferred growth in the in-plane direction of the conjugated backbone in the aggregates. On the other hand, the similar line shape of $\varepsilon^{\prime \prime}{ }_{\text {in-plane }}$ and $\varepsilon^{\prime \prime}{ }_{\text {out-of-plane }}$ in MAPLE-deposited samples indicates no preference in the growth direction of the aggregates, providing further evidence that aggregate formation is not impacted by interactions with the substrate.

Finally, we would like to point out that since MAPLE films are formed from merging of discrete globular units overlapping one another, it is possible that only the near-substrate layer would exhibit substrate-sensitive morphological differences. Thus, we expect to see a stronger substrate dependence behavior upon decreasing the film thickness towards the globule size, because self-assembled monolayers (SAMs) such as OTS are known to have localized effect on polymer structure. ${ }^{45} \mathrm{~A}$ thorough examination on film forming mechanism therefore should motivate a thickness dependence study of polymer 
structure on different substrates and will be the subject of our investigations in the near future.

\section{CONCLUSIONS}

In this work, we compared molecular structure of MAPLE-deposited P3HT films and spin-casted P3HT films on 3 different substrates: OTS, $\mathrm{SiO}_{2}$ and PEDOT. GIWAXS data showed higher degrees of disorder and a more random orientation of polymer crystallites in all MAPLE-deposited samples compared to spincast counterparts. Additionally, VASE measurements revealed little overall preferential orientation of the conjugated polymer backbone in MAPLE films, further highlighting the highly disordered structure in films deposited by the MAPLE technique. Partial pole figures from GIWAXS measurements indicated a negligible dependence of average polymer morphology on substrate chemistry of MAPLE-deposited films compared to the spincast analogs. MAPLE-deposited samples exhibited identical average structures on all substrates, whereas spin-cast samples prepared on PEDOT and OTS showed higher degrees of crystallization and more edge-on orientations of polymer crystallites than the sample prepared on $\mathrm{SiO}_{2}$. The implications of the morphology on charge transport were illustrated with temperature dependent studies of field-effect mobilities, yielding results suggesting that structural disorder or unfavorable molecular orientations revealed by GIWAXS and VASE may not be the limiting factors for in-plane carrier transport. These results also supported our proposed mechanism of the film formation in MAPLE films, specifically, that the aggregates nucleate and form prior to reaching the substrate. Future efforts involving manipulation of molecular order in MAPLE films will focus on factors such as controlling the degree of $\pi$ stacked aggregates in the emulsion rather than changing the chemistry of the underlying substrates.

\section{ACKNOWLEDGEMENTS}

B.X.D acknowledges partial financial support from the University of Michigan and Vietnam Education Foundation. We acknowledge partial support from the National Science Foundation (NSF), Division of Materials Research (DMR-1305749). The authors thank Saeed Ahmadi Vaselabadi from Stein Group for compiling the GIWAXS raw detector images. G.E.S. acknowledges financial support from the National Science Foundation under Grant No. DMR-1151468. Use of the Advanced Photon Source, an Office of Science User Facility operated for the U.S. Department of Energy (DOE) by Argonne National Laboratory, was supported by the U.S. DOE under Contract No. DE-AC02-06CH11357.

\section{REFERENCES AND NOTES}

(1) Sirringhaus, H. Adv. Mater. 2014, 26 (9), 1319-1335.

(2) Geffroy, B.; le Roy, P.; Prat, C. Polym. Int. 2006, 55 (6), 572-582.

Müllen, K.; Scherf, U. Organic Light Emitting Devices: Synthesis, Properties and Applications; Wiley: New York, 2006.

(4) Günes, S.; Neugebauer, H.; Sariciftci, N. S. Chem. Rev. 2007, 107 (4), 1324-1338.

(5) Kroon, R.; Lenes, M.; Hummelen, J. C.; Blom, P. W. M.; de Boer, B. Polym. Rev. 2008, 48 (3), 531-582.

(6) Giebink, N. C.; Forrest, S. R. Phys. Rev. B 2008, 77 (23), 1-9.

(7) Newman, C. R.; Frisbie, C. D.; Demetrio, A.; Filho, S.; Bre, J. 2004, 4436-4451.

(8) A. Pivrikas, N. S. Sariciftci, G. Juška, R. Ö. Prog. PHOTOVOLTAICS Res. Appl. 2007, 15, 677-696.

(9) Mcgi, R. A.; Chriseya, D. B.; Piqué, A.; Misnac, T. E.; Washington, D. C. Laser Appl. Microelectron. Optoelectron. Manuf. III 1998, 3274, 255-266.

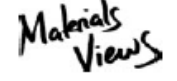

WWW.MATERIALSVIEWS.COM 
(10) Piqué, a.; McGill, R. a.; Chrisey, D. B.; Leonhardt, D.; Mslna, T. E.; Spargo, B. J.; Callahan, J. H.; Vachet, R. W.; Chung, R.; Bucaro, M. a. Thin Solid Films 1999, 355, 536-541.

(11) Chrisey, D. B.; Piqué, A.; McGill, R. A.; Horwitz, J. S.; Ringeisen, B. R.; Bubb, D. M.; Wu, P. K. Chem. Rev. 2003, 103 (2), 553-576.

(12) Shepard, K. B.; Priestley, R. D. Macromol. Chem. Phys. 2013, 214 (8), 862-872.

(13) Guo, Y.; Morozov, A.; Schneider, D.; Chung, J. W.; Zhang, C.; Waldmann, M.; Yao, N.; Fytas, G.; Arnold, C. B.; Priestley, R. D. Nat. Mater. 2012, 11 (4), 337-343.

(14) Shepard, K. B.; Guo, Y.; Arnold, C. B.; Priestley, R. D. Appl. Phys. A Mater. Sci. Process. 2013, 110 (4), 771-777.

(15) Sima, F.; Axente, E.; Ristoscu, C.; Mihailescu, I. N.; Kononenko, T. V.; Nagovitsin, I. A.; Chudinova, G.; Konov, V. I.; Socol, M.; Enculescu, I.; Sima, L. E.; Petrescu, S. M. J. Biomed. Mater. Res. Part A 2011, 96 A (2), 384-394.

(16) Casey, C. N.; Campbell, S. E.; Gibson, U. J. Biosens. Bioelectron. 2010, 26 (2), 703709.

(17) Cristescu, R.; Popescu, C.; Socol, G.; Visan, A.; Mihailescu, I. N.; Gittard, S. D.; Miller, P. R.; Martin, T. N.; Narayan, R. J.; Andronie, A.; Stamatin, I.; Chrisey, D. B. Appl. Surf. Sci. 2011, 257 (12), 52875292.

(18) Li, A.; Dong, B. X.; Green, P. MRS Commun. 2015, 5 (04), 593-598.

(19) Gutiérrez-Llorente, a.; Horowitz, G.; Pérez-Casero, R.; Perrière, J.; Fave, J. L.; Yassar, a.; Sant, C. Org. Electron. physics, Mater. Appl. 2004, 5 (1-3), 29-34.

(20) McCormick, R. D.; Lenhardt, J.; Stiff-
Roberts, A. D. Polymers (Basel). 2012, 4 (4), 341-354.

(21) Pate, R.; Lantz, K. R.; Stiff-Roberts, A. D. IEEE J. Sel. Top. Quantum Electron. 2008, $14(4), 1022-1030$.

(22) Ge, W.; Li, N. K.; McCormick, R. D.; Lichtenberg, E.; Yingling, Y. G.; StiffRoberts, A. D. ACS Appl. Mater. Interfaces 2016, 8 (30), 19494-19506.

(23) Mariano, F.; Caricato, A. P.; Accorsi, G.; Leo, C.; Cesaria, M.; Carallo, S.; Genco, A.; Simeone, D.; Tunno, T.; Martino, M.; Gigli, G.; Mazzeo, M. J. Mater. Chem. C 2016.DOI: 10.1039/C6TC01826B

(24) Ge, W.; McCormick, R. D.; Nyikayaramba, G.; Stiff-Robertsa, A. D. Appl. Phys. Lett. 2014, 104 (22).

(25) Caricato, a. P.; Cesaria, M.; Gigli, G.; Loiudice, a.; Luches, a.; Martino, M.; Resta, V.; Rizzo, a.; Taurino, a. Appl. Phys. Lett. 2012, 100 (7), 073306.

(26) Ge, W.; Atewologun, A.; Stiff-Roberts, A. D. Org. Electron. physics, Mater. Appl. 2015, 22, 98-107.

(27) Jiang, Z.; Li, X.; Strzalka, J.; Sprung, M.; Sun, T.; Sandy, A. R.; Narayanan, S.; Lee, D. R.; Wang, J. J. Synchrotron Radiat. 2012, 19 (4), 627-636.

(28) Vaselabadi, S. A.; Shakarisaz, D.; Ruchhoeft, P.; Strzalka, J.; Stein, G. E. J. Polym. Sci. Part B Polym. Phys. 2016, 113.

(29) Jiang, Z. J. Appl. Crystallogr. 2015, 48 (3), 917-926.

(30) Rivnay, J.; Noriega, R.; Kline, R. J.; Salleo, A.; Toney, M. F. Phys. Rev. B - Condens. Matter Mater. Phys. 2011, 84 (4), 1-20.

(31) Baker, J. L.; Jimison, L. H.; Mannsfeld, S.; Volkman, S.; Yin, S.; Subramanian, V.; Salleo, A.; Alivisatos, A. P.; Toney, M. F. 
Langmuir 2010, 26 (11), 9146-9151.

(32) Campoy-Quiles, M.; Alonso, M. I.;

Bradley, D. D. C.; Richter, L. J. Adv. Funct.

Mater. 2013, 24, 2116-2134.

(33) Brinkmann, M.; Wittmann, J.-C. Adv.

Mater. 2006, 18 (7), 860-863.

(34) Rivnay, J.; Noriega, R.; Northrup, J. E.; Kline, R. J.; Toney, M. F.; Salleo, A. Phys.

Rev. B - Condens. Matter Mater. Phys. 2011, 83 (12), 1-4.

(35) Zhang, X.; Bronstein, H.; Kronemeijer, A. J.; Smith, J.; Kim, Y.; Kline, R. J.; Richter, L. J.; Anthopoulos, T. D.; Sirringhaus, H.; Song, K.; Heeney, M.; Zhang, W.; McCulloch, I.; Delongchamp, D. M. Nat. Commun. 2013, 4, 2238.

(36) Prosa, T. J.; Moulton, J.; Heeger, a. J.; Winokur, M. J. Macromolecules 1999, 32 (12), 4000-4009.

(37) Brinkmann, M.; Rannou, P. Adv. Funct. Mater. 2007, 17 (1), 101-108.

(38) Wu, Z.; Petzold, A.; Henze, T.; ThurnAlbrecht, T.; Lohwasser, R. H.; Sommer, M.; Thelakkat, M. Macromolecules 2010, 43 (10), 4646-4653.

(39) Joshi, S.; Pingel, P.; Grigorian, S.; Panzner, T.; Pietsch, U.; Neher, D.; Forster, M.; Schere, U. Macromolecules 2009, 42 (13), 4651-4660.

(40) Joshi, S.; Joshi, S.; Grigorian, S.; Grigorian, S.; Pietsch, U.; Pietsch, U.; Pingel, P.; Pingel, P.; Zen, A.; Zen, A.; Neher, D.; Neher, D.; Scherf, U.; Scherf, U. Macromolecules 2008, 41 (18), 68006808.

(41) Chang, J.-F.; Sun, B.; Breiby, D. W.; Nielsen, M. M.; Sölling, T. I.; Giles, M.; McCulloch, I.; Sirringhaus, H. Chem. Mater. 2004, 16 (23), 4772-4776.

(42) Brinkmann, M.; Rannou, P.
Macromolecules 2009, 42, 1125-1130.

Mannsfeld, S. C. B.; Virkar, A.; Reese, C.; Toney, M. F.; Bao, Z. Adv. Mater. 2009, 21 (22), 2294-2298.

(44) Hammond, M. R.; Kline, R. J.; Herzing, A. a; Richter, L. J.; Germack, D. S.; Ro, H.W.; Soles, C. L.; Fischer, D. a; Xu, T.; Yu, L.; Toney, M. F.; Delongchamp, D. M. ACS Nano 2011, 5 (10), 8248-8257.

(45) Jimison, L. H.; Himmelberger, S.; Duong, D. T.; Rivnay, J.; Toney, M. F.; Salleo, A. J. Polym. Sci. Part B Polym. Phys. 2013, 51 (7), 611-620.

(46) Dong, B. X.; Huang, B.; Tan, A.; Green, P. F. J. Phys. Chem. C 2014, 118 (31), 17490-17498.

(47) Gurau, M. C.; Delongchamp, D. M.; Vogel, B. M.; Lin, E. K.; Fischer, D. a; Sambasivan, S.; Richter, L. J. Langmuir 2007, 23 (2), 834-842.

(48) Clark, J.; Silva, C.; Friend, R.; Spano, F. Phys. Rev. Lett. 2007, 98 (20), 206406.

(49) DeLongchamp, D. M.; Kline, R. J.; Fischer, D. a; Richter, L. J.; Toney, M. F. Adv. Mater. 2011, 23 (3), 319-337.

(50) Leveugle, E.; Zhigilei, L. V. J. Appl. Phys. 2007, 102 (7), 1-19.

(51) Zhang, X.; Hudson, S. D.; Delongchamp, D. M.; Gundlach, D. J.; Heeney, M.; McCulloch, I. Adv. Funct. Mater. 2010, 20 (23), 4098-4106.

(52) Kline, R. J.; Mcgehee, M. D.; Kadnikova, E. N.; Liu, J.; Fre, J. M. J.; Toney, M. F. Macromolecules 2005, 3312-3319.

(53) Dinelli, F.; Murgia, M.; Levy, P.; Cavallini, M.; Biscarini, F.; De Leeuw, D. M. Phys. Rev. Lett. 2004, 92 (11), 116802-1.

(54) Nielsen, C. B.; Turbiez, M.; McCulloch, I. Adv. Mater. 2013, 25 (13), 1859-1880. 
(55) Zhang, W.; Smith, J.; Watkins, S. E.; Gysel, R.; McGehee, M.; Salleo, A.; Kirkpatrick, J.; Ashraf, S.; Anthopoulos, T.; Heeney, M.; McCulloch, I. J. Am. Chem. Soc. 2010, 132 (33), 1143711439.

(56) Noriega, R.; Rivnay, J.; Vandewal, K.; Koch, F. P. V; Stingelin, N.; Smith, P.; Toney, M. F.; Salleo, A. Nat. Mater. 2013, 12 (11), 1038-1044.
(57) Himmelberger, S.; Salleo, A.; Science, M. MRS Commun. 2015, 1-13.

(58) Shepard, K. B.; Arnold, C. B.; Priestley, R. D. Appl. Phys. Lett. 2013, 103 (12), 123105.

(59) Shepard, K. B.; Arnold, C. B.; Priestley, R. D. ACS Macro Lett. 2014, 3 (10), 10461050. 


\section{GRAPHICAL ABSTRACT}

BAN XUAN DONG, ANTON LI, JOSEPH STRZALKA, GILA E. STEIN, PETER F. GREEN

\section{MOLECULAR ORGANIZATION IN MAPLE-DEPOSITED CONJUGATED POLYMER THIN FILMS AND THE IMPLICATIONS FOR CARRIER TRANSPORT CHARACTERISTICS.}

Molecular order in poly(3-hexylthiophene) (P3HT) films deposited using the novel vapor-assisted deposition technique Matrix Assisted Pulsed Laser Evaporation (MAPLE) was investigated. Unlike the film prepared using conventional spin-casting methods, the structure of MAPLE-deposited films is insensitive to the substrate chemistries. These films possess a larger degree of random crystallite orientations. The seemingly unfavorable morphology of MAPLE-deposited samples however do not have appear to have detrimental effects on in-plane transport characteristics, suggesting that field-effect carrier transport in conjugated polymer devices is resilient to structure.

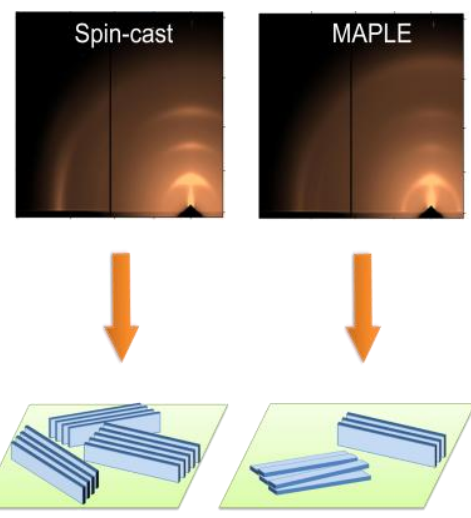

